\title{
Análises das causas das subnotificações das intoxicações por agrotóxicos na rede de saúde em município do Sul do Brasil
}

\author{
Analysis of the causes of pesticide poisoning underreporting \\ in the healthcare network in a municipality in Southern Brazil
}

Eleonora Escobar Tosetto ((http://orcid.org/0000-0002-6344-9134) ${ }^{1}$

Antônio Inácio Andrioli ((http://orcid.org/0000-0002-3406-3953) ${ }^{2}$

Pedro Ivan Christoffoli ((http://orcid.org/0000-0002-1956-2674) ${ }^{2}$

${ }^{1}$ Instituto São José. Rua Barão do Rio Branco 2460, Centro. 85301-030 Laranjeiras do Sul PR Brasil. eleonora.tosetto@ portalser.org

${ }^{2}$ Programa de Pós-

Graduação em Agroecologia

e Desenvolvimento Rural Sustentável, Universidade Federal da Fronteira Sul. Laranjeiras do Sul SC Brasil.

\begin{abstract}
The intensive use of pesticides in agriculture is associated with several negative externalities, which affect both the environment and public health. It is known that the number of records is lower than that of pesticide poisonings, and that underreporting occurs. Aiming to understand this underreporting, we analyzed the data from two information systems for notifications and health injuries and a case study was carried out with professionals from the $5^{\text {th }}$ Regional Health Center and the health network of a municipality in the state of Paraná. It was concluded that underreporting occurs in the information systems, confirming the inconsistency of data and the lack of communication between them, making it difficult to understand the reality of poisonings. In the case study, the occurrence of prior underreporting was identified: when many workers do not seek health systems; the training of professionals is insufficient and neutralized by high turnover, especially among physicians; the diagnosis, conduct and treatment of pesticide poisoning are not part of the curriculum of many courses in the health area; the capacity of health services in many municipalities falls short of the demand, causing work overload to the professionals and the consequent underreporting.
\end{abstract}

Key words Pesticides, Poisoning, Public health, Toxic contamination, Health surveillance
Resumo O uso intensivo de agrotóxicos na agricultura está associado a externalidades negativas, que afetam o meio ambiente e a saúde pública. Sabe-se que o número de registros é menor que os de intoxicações por pesticidas, ocorrendo subnotificações. Com o objetivo de compreender esse evento, analisou-se os dados de dois sistemas de informação de notificações e agravos à saúde e realizou-se estudo de caso com profissionais da $5^{a}$ Regional de Saúde e da rede de saúde de um município do Paraná. Confirmou-se que ocorrem as subnotificações nos sistemas de informações, a inconsistência de dados e a falta de comunicação entre eles, dificultando a compreensão da realidade das intoxicações. No estudo de caso identificouse a ocorrência de subnotificação prévia: quando muitos trabalhadores não procuram os sistemas de saúde; as capacitações dos profissionais são insuficientes e neutralizadas pela grande rotatividade, especialmente de médicos; o diagnóstico, a conduta e o tratamento das intoxicações por agrotóxicos não fazem parte do currículo de muitos dos cursos da área de saúde; e a capacidade dos serviços de saúde de muitos municípios está aquém da demanda, ocasionando sobrecarga de trabalho aos profissionais e a consequente subnotificação.

Palavras-chave Agrotóxicos, Intoxicações, Saúde pública, Envenenamento, Vigilância em saúde 


\section{Introdução}

A agricultura brasileira passou por um processo de modernização conservadora a partir dos anos 1950, buscando o aumento da produção e produtividade mediante a mecanização e uso de adubos sintéticos e agrotóxicos. O crédito rural subsidiado e a assistência técnica estatal foram os principais mecanismos de indução do uso de agrotóxicos $^{1}$ a que hoje se soma a força privada de vendas das empresas ${ }^{2}$. O uso intensivo desses produtos está associado a externalidades negativas, que impactam o meio ambiente e a saúde pública. Há efeitos diretos sobre trabalhadores e outros moradores do meio rural, e indiretos sobre a população urbana via contaminação de alimentos, água potável e mesmo da chuva ${ }^{3,4,5}$. Considerando a amplitude do setor agrícola, a realidade das intoxicações se estende por todo território nacional ${ }^{6}$. A real dimensão dessa epidemia é desconhecida. A subnotificação tem contribuído para ocultar a necessidade premente de ações de vigilância e impedir o acesso dos trabalhadores aos seus direitos e à sua saúde.

Utiliza-se o termo subnotificação quando algo é notificado abaixo da realidade, produzindo um silêncio epidemiológico em regiões onde sabidamente existem ocorrência do agravo ${ }^{7}$. Esses fatos dificultam a detecção precoce ou, na maioria das vezes, o reconhecimento da síndrome clínica. A Organização Mundial da Saúde (OMS) reconhece que a subnotificação das intoxicações por agrotóxicos é da ordem de 1:50 para cada caso registrado ${ }^{8}$. O presente artigo busca compreender a subnotificação das intoxicações na visão dos profissionais de saúde, a fim de auxiliar na elucidação desse fenômeno que contribui para o desconhecimento da gravidade do problema.

\section{Produção agrícola e consumo de agrotóxicos no Brasil e no Estado do Paraná}

O Brasil é um dos maiores consumidores de agrotóxicos em números absolutos ${ }^{4,6,8}$. Utilizados para a proteção de cultivos e criações esses pesticidas são, contudo, agressivos à saúde humana 9 . O consumo de agrotóxicos no Brasil alcança $33,1 \%$ dos agricultores (1,68 milhões de estabelecimentos). Esse número foi $20 \%$ maior do que no censo de 2006. Dos estabelecimentos que usaram pesticidas, $16 \%$ dos responsáveis não sabiam ler e escrever e, destes, $89 \%$ não recebeu orientação técnica. Dos 70\% que tinham no máximo o ensino fundamental (1.170.784), apenas $31 \%$ declararam ter recebido orientação técnica ${ }^{10}$, caracteri- zando uma grave situação na ponta do consumo. O Estado do Paraná é o segundo maior produtor de grãos (16,23\% do total na safra 2017/2018) e o terceiro maior consumidor de agrotóxicos do Brasil, com um volume total de 92.398 toneladas em 2017 representando um consumo médio de 8,08 quilos por habitante/ano ${ }^{11-13}$.

$\mathrm{O}$ estudo deste artigo foi realizado no município de Laranjeiras do Sul, localizado na $5^{a}$ Regional de Saúde, uma das cinco regionais que mais comercializam agrotóxicos no estado, consumindo 31.554 toneladas no período 2014$2017^{11}$. O município de Laranjeiras do Sul tem característica agrícola, com produção principal de soja, milho e trigo. O crédito rural no município variou entre 2013 e 2015 de $\mathrm{R} \$ 72,8$ a 98,2 milhões $^{13}$. Esse recurso impulsiona o consumo de agrotóxicos. Em Laranjeiras foram utilizados 6,84 quilogramas/habitante $(\mathrm{Kg} / \mathrm{hab})$ em 2013 e 7,40 em 2018. No entorno existem municípios dependentes da sua rede assistencial hospitalar. Em 2013 alguns deles também alcançaram elevado consumo de agrotóxicos por habitante/ano, como em Cantagalo (47,39 Kg/hab), Porto Barreiro $(25,52 \mathrm{Kg} / \mathrm{hab})$ e Virmond $(116,59 \mathrm{Kg} / \mathrm{hab})$. Em 2018 o consumo ainda foi bastante significativo: Cantagalo (14,21 kg/hab), Porto Barreiro $(38,57 \mathrm{Kg} / \mathrm{hab})$ eVirmond $(29,44 \mathrm{~kg} / \mathrm{hab})$ apresentam médias muito superiores à nacional ${ }^{11,14}$. Portanto, seria esperado que a rede de saúde de Laranjeiras estivesse preparada para a notificação de eventuais casos de intoxicação.

\section{Agrotóxicos, intoxicações e impactos na saúde humana}

Os agrotóxicos impactam a saúde humana e ambiental, produzindo efeitos que variam conforme o princípio ativo, a dose absorvida e a forma de exposição ${ }^{15}$. Essa toxicidade depende das propriedades dos ingredientes ativos e coadjuvantes do produto, podendo levar a óbito ${ }^{16}$. A exposição aos agrotóxicos pode ocorrer a partir do contato com a pele, mucosas, pela respiração e pela ingestão de alimentos contaminados. A exposição ocupacional ocorre durante a diluição, a preparação da calda, durante e após a aplicação dos produtos ${ }^{16,17}$. Pode ainda ocorrer a deriva dos agrotóxicos para fora da área de aplicação. Também são registradas intoxicações de familiares, no caso de lavagem de roupas usadas na aplicação $0^{18}$.

As intoxicações podem ser agudas ou crônicas. Intoxicação aguda é a que ocasiona alteração no estado de saúde de um ou mais indivíduos, 
resultante da interação nociva de uma substância com o organismo vivo. Ela pode ocorrer de forma leve, moderada ou grave, dependendo da quantidade de veneno absorvido, do tempo de absorção, da toxicidade do produto entre outros. Manifesta-se por meio de um conjunto de sinais e sintomas que podem ocorrer de forma súbita, minutos ou horas após a exposição. Geralmente é única e ocorre no período de até 24 horas ${ }^{19}$.

Intoxicações crônicas representam a alteração no estado de saúde de um ou mais indivíduos, resultante da interação nociva a uma substância. Ela surge no decorrer de repetidas exposições ao toxicante durante longos períodos, incluindo acumulação de danos genéticos, resultando em quadros clínicos indefinidos, confusos e muitas vezes irreversíveis. Nesse caso, os diagnósticos são difíceis de serem estabelecidos e há uma maior dificuldade na associação causa/efeito, principalmente quando há exposição do paciente a múltiplos produtos ${ }^{19-22}$. A atuação dos profissionais na identificação dos sintomas, caracterização, encaminhamentos e registros é primordial para se ter uma base de dados confiável.

Há dificuldade em associar patologias como câncer, malformações, alergias, depressão, Parkinson e outras doenças crônicas, ao uso de agrotóxi$\cos ^{23}$. Nessa condição, passam despercebidos produtos como o Glifosato, responsável pela metade dos agrotóxicos consumidos no Brasil, mas classificado como de baixa toxidez aguda e provavelmente carcinogênico para humanos pela Agência Internacional de Pesquisas do Câncer (IARC) ${ }^{8,9}$. Assim também ocorre com carbamatos e outros produtos causadores de depressão e indutores de ideação de suicídio ${ }^{18}$.

\section{Subnotificações de intoxicações por agrotóxicos na rede de saúde}

Notificação é a comunicação da ocorrência de determinada doença ou agravo à saúde à autoridade sanitária por profissionais de saúde ou qualquer cidadão, para fins de adoção de medidas de intervenção pertinentes. Historicamente, a notificação compulsória tem sido a principal fonte da vigilância epidemiológica, a partir da qual geralmente se desencadeia o processo informação-decisão-ação ${ }^{24}$.

As ações de monitoramento de intoxicações por agrotóxicos em âmbito nacional iniciaram na década de 1980, pelo Sistema Nacional de Informações Tóxico Farmacológicas (Sinitox), constituído pelo Ministério da Saúde e vinculado à Fundação Oswaldo Cruz ${ }^{25}$ (Fiocruz) e o
Sistema de Informações de Agravos de Notificação (Sinan) tem como objetivo de registrar e processar os dados sobre agravos de notificação em todo território nacional e é feito de forma compulsória (Quadro 1). Sua finalidade foi coordenar a coleta, a compilação, a análise e a divulgação dos casos de intoxicação e envenenamento notificados no país". Os principais sistemas de notificação de agravos por intoxicações por agrotóxicos estão resumidos no Quadro 1.

Além desses, é possível obter dados sobre intoxicações no Sistema de Informações sobre Nascidos Vivos (SINASC - dados de malformação fetal), ligado ao Ministério da Saúde, e no Cadastro de Acidentes de Trabalho (CAT), do Ministério do Trabalho. Logo, existem vários sistemas oficiais que registram intoxicações por agrotóxicos, contudo, nenhum deles responde adequadamente como instrumento de vigilância deste tipo de agravo $^{21}$.

As notificações das intoxicações de pacientes expostos a agrotóxicos são de responsabilidade da vigilância em saúde, tendo como atribuições a coleta e processamento de dados, e análise e interpretação de informações, para recomendar medidas de controle apropriadas a cada região e avaliar se as mesmas são efetivas e eficientes ${ }^{6}$. O cumprimento dessas funções depende da disponibilidade de informações para subsidiar o desencadeamento de ações. A qualidade da informação depende da adequada coleta dos dados que são gerados no local dos agravos e facilita o planejamento, avaliação, manutenção e aprimoramento das ações de prevenção em saúde ${ }^{26}$.

A informação depende da capacitação e motivação, preparando os profissionais responsáveis para diagnosticar e notificar corretamente e realizar a investigação epidemiológica com confiabilidade $^{27}$. O Protocolo de Avaliação das Intoxicações Crônicas por Agrotóxicos ${ }^{19}$ reconhece que os profissionais da saúde no Brasil carecem de instrumentos clínicos para orientar o diagnóstico das intoxicações crônicas e de critérios para definir o estabelecimento da relação dessas intoxicações com o trabalho e o ambiente. A subnotificação também implica na invisibilidade dos custos dessas intoxicações para o Sistema Único de Saúde (SUS). Isso afeta a implantação de políticas públicas, que amparem tanto o agricultor como a sociedade ${ }^{28}$.

A análise das causas de subnotificação revela um quadro complexo. Mesmo que exista a iniciativa da notificação, não há suporte para sua materialização ${ }^{7}$. Além da dificuldade de identificar e diagnosticar as intoxicações, há complicadores 
Quadro 1. Brasil. Sistemas de informações de notificações de agravos por intoxicações por agrotóxicos.

\begin{tabular}{|c|c|c|c|}
\hline $\begin{array}{c}\text { Sistema de } \\
\text { Coleta de Dados }\end{array}$ & Sigla/ Site & Finalidade & $\begin{array}{c}\text { Responsáveis pelo } \\
\text { preenchimento dos dados }\end{array}$ \\
\hline $\begin{array}{l}\text { Sistema Nacional } \\
\text { de Informações } \\
\text { Tóxico } \\
\text { Farmacológicas }\end{array}$ & $\begin{array}{l}\text { SINITOX - http:// } \\
\text { sinitox.icict. } \\
\text { fiocruz.br/ }\end{array}$ & $\begin{array}{l}\text { Coordenar a coleta, a compilação, a } \\
\text { análise e a divulgação dos casos de } \\
\text { intoxicação e envenenamento notificados } \\
\text { no país }\end{array}$ & $\begin{array}{l}\text { Ministério da Saúde e } \\
\text { Fundação Osvaldo Cruz } \\
\text { (Fiocruz). Rede Nacional } \\
\text { de Centros de Informação } \\
\text { e Assistência Toxicológica } \\
\text { (Renaciat), composta por } \\
\text { diversas unidades presentes } \\
\text { em todas as regiões do Brasil }\end{array}$ \\
\hline $\begin{array}{l}\text { Sistema de } \\
\text { Informações } \\
\text { de Agravos de } \\
\text { Notificação }\end{array}$ & $\begin{array}{l}\text { SINAN - http:// } \\
\text { sinan.saude.gov. } \\
\text { br/sinan/login/ } \\
\text { login.jsf }\end{array}$ & $\begin{array}{l}\text { Tem como objetivo o registro e o } \\
\text { processamento dos dados sobre agravos } \\
\text { de notificação em todo território } \\
\text { nacional. Fornece informações para } \\
\text { análise do perfil de morbidade e } \\
\text { contribuindo, dessa forma, para a tomada } \\
\text { de decisões em nível municipal, estadual e } \\
\text { federal. De notificação compulsória }\end{array}$ & $\begin{array}{l}\text { Ministério da Saúde (MS). } \\
\text { Unidade básica saúde, } \\
\text { Secretaria Saúde e Serviços } \\
\text { de informação e/ou } \\
\text { vigilância epidemiológica }\end{array}$ \\
\hline $\begin{array}{l}\text { Sistema de } \\
\text { Informações de } \\
\text { Mortalidade }\end{array}$ & $\begin{array}{l}\text { SIM - http://sim. } \\
\text { saude.gov.br/ } \\
\text { default.asp }\end{array}$ & $\begin{array}{l}\text { Tem a finalidade de reunir dados } \\
\text { quantitativos, qualitativos e regulares } \\
\text { sobre a mortalidade no país. A Declaração } \\
\text { de Óbito (DO) deve ser preenchida pelo } \\
\text { médico que atendeu, ou em sua ausência, } \\
\text { por duas pessoas qualificadas, que } \\
\text { tenham verificado a morte }\end{array}$ & $\begin{array}{l}\text { Secretaria de Saúde } \\
\text { municipal / estadual }\end{array}$ \\
\hline $\begin{array}{l}\text { Sistema de } \\
\text { Informações } \\
\text { Hospitalares }\end{array}$ & $\begin{array}{l}\text { SIH - http:// } \\
\text { datasus.saude. } \\
\text { gov.br/sistemas- } \\
\text { e-aplicativos/ } \\
\text { hospitalares / } \\
\text { sihsus }\end{array}$ & $\begin{array}{l}\text { Armazena os dados sobre as internações } \\
\text { hospitalares no âmbito do SUS, } \\
\text { informado mensalmente por todos os } \\
\text { estabelecimentos de saúde públicos, } \\
\text { conveniados e contratados, que realizam } \\
\text { internações e consolidados pelos } \\
\text { municípios e pelos estados }\end{array}$ & Hospitais e clínicas médicas \\
\hline
\end{tabular}

Fonte: DATASUS ${ }^{35}$, Fiocruz ${ }^{25}$, Ministério da Saúde ${ }^{36}$.

de ordem metodológica, analítica e estrutural, que contribuem mundialmente para a imprecisão dos dados disponíveis ${ }^{29}$.

A notificação é habitualmente realizada de modo precário, devido ao desconhecimento de sua importância, ao descrédito dos serviços de saúde, à falta de acompanhamento e supervisão da rede de serviços e de retorno dos dados coletados e das ações que foram geradas pela análise $\mathrm{e}^{7}$. Para diminuir as subnotificações indica-se a padronização de fluxos de atendimento, a elaboração de materiais informativos com procedimentos de saúde do trabalhador e capacitações periódicas aos profissionais e agricultores ${ }^{30}$.

Estudos demonstram que o aumento das notificações devido à intensificação do uso de agrotóxicos não motivou ações mais efetivas de vigilância em saúde. Interesses diversos e contraditórios no aparato estatal obstaculizam a proteção à saúde coletiva ${ }^{31}$. As subnotificações pioram esse cenário, ao invisibilizar intoxicações e contribuir para a não atuação da vigilância em saúde dos trabalhadores rurais.

Entretanto, dúvidas sobre o grau de intoxicação existente no país não deveriam impedir a imediata tomada de ação pelos órgãos públicos. A caracterização do risco, com evidências abundantes, indica a probabilidade de dano. Não é necessário comprovar a ocorrência de agravos para desencadear políticas públicas de promoção e proteção da saúde ${ }^{4}$. Contudo, o cenário de controvérsia científica é utilizado como arma, por grupos de interesses econômicos, que incidem ativamente sobre o Estado e as políticas públicas. 


\section{Métodos}

A pesquisa consistiu em estudo de caso tipo survey, de caráter exploratório, transversal, e com abordagem quali-quantitativa. Baseou-se em aplicação de questionários diferenciados entre segmentos profissionais e níveis do sistema de saúde, com perguntas fechadas e abertas, utilizando-se amostragem não probabilística estratificada, por adesão, com trabalhadores de vários níveis do sistema de saúde e diversas categorias profissionais, e aplicados no período de novembro de 2015 a março de 2016. Ainda, efetuou-se pesquisa documental e bibliográfica, e comparação dos dados secundários dos sistemas de saúde Sinitox e Sinan.

As entrevistas foram feitas com profissionais vinculados às Secretarias de Saúde em nível estadual, na $5^{\text {a }}$ Regional e na Secretaria Municipal, além de profissionais de postos de saúde e dos dois hospitais que prestam atendimento a Urgências e Emergências do município de Laranjeiras do Sul (PR). Ao todo participaram 45 respondentes, sendo nove médicos, dez enfermeiros, 15 técnicos e auxiliares de enfermagem, sete gestores do sistema de saúde e quatro outros profissionais. Dos entrevistados, 35 atuavam na linha de frente (atendimento direto), sete no nível de gestão do sistema e três desempenhavam outras funções acessórias.

Os resultados identificados devem ser tomados como indicadores acerca do tema, não devendo ser generalizados para outras situações e contextos podendo, contudo, trazer inferências úteis. Algumas respostas podem ter espelhado fatores como a preocupação de autoproteção dos profissionais frente às suas habilitações legais no tema. Esclarecidos esses aspectos, os resultados contribuem para uma melhor percepção do problema.

\section{Resultados e discussão}

Primeiramente analisaram-se os resultados dos sistemas de informações e agravos das intoxicações por agrotóxicos. A segunda parte do estudo buscou compreender, de forma exploratória, o ponto de vista de profissionais da saúde sobre as possíveis causas das subnotificações.

\section{Análise dos sistemas de informação de notificação de agravos por intoxicações por agrotóxicos}

Os dados de intoxicação humana por agrotóxicos registrados nos sistemas de informações de notificações e agravos demonstraram várias inconformidades e inconsistências. Inicialmente compararam-se os dados do Sinitox nas principais regiões produtoras do Paraná com os dados do SINAN (2013-2017). Observou-se que o Centro de Informação Toxicológica de Cascavel, que consolida dados de diversos municípios, inclusive Laranjeiras do Sul, não registrou nenhuma notificação de intoxicações no período. $\mathrm{O}$ mesmo se deu em todas as outras cidades polo, responsáveis pela consolidação de dados no estado do Paraná, segundo o Sinitox (Tabela 1).

O Sinan, de notificação obrigatória na atenção básica de saúde, no mesmo período, registrou centenas de casos de intoxicações no Paraná. O contraste de dados consolida a visão de que o Sinitox, é pouco confiável, sujeito a lacunas de comunicação e registro, contribuindo para alimentar a crítica de que os dados oficiais brasileiros sobre intoxicações por agrotóxicos não retratam a realidade. Seriam insuficientes, parciais, fragmentados, desarticulados e dispersos em várias fontes de dados ${ }^{21,7}$. Entretanto, cabe avaliar se as notificações registradas no Sinan são conformes a realidade.

É dever do Estado e direito da cidadania identificar a real dimensão das intoxicações no país, visto que, além dos dramas humanos envolvidos, há impacto sobre custos na saúde pública oriundos do tratamento. As vendas de agrotóxicos no Brasil têm impostos estaduais reduzidos e benefícios na carga fiscal federal. A estranha combinação de eficiência de um lado (benefícios ao setor) e ineficiência de outro (na fiscalização de seus efeitos deletérios), reflete relações de poder político inerentes à questão agrária em nosso país ${ }^{1,4}$. Há uma externalização negativa dos custos das perdas de vidas e dos gastos em saúde nos preços dos insumos utilizados ${ }^{32}$ pelo agronegócio. A deterioração da qualidade ambiental, os altos custos de despoluição e de tratamento de saúde não são incluídos no preço de produção agrícola. Já à produção agroecológica exige-se selo de conformidade orgânica sobrecarregando custos aos pequenos agricultores que produzem alimentos limpos, ao passo que franqueia livre acesso aos mercados aos produtos contaminados.

Essa falha no sistema de impostos e preços, caracteriza subsídio ao modelo destrutivo do 
agronegócio. A gratuidade da contaminação ambiental e das intoxicações decorrentes contribui para a continuidade de ocorrência de tão grave prática social e elevada degradação do meio.

\section{A percepção de profissionais acerca da notificação das intoxicações por agrotóxicos}

A pesquisa buscou analisar a percepção de profissionais de saúde visando identificar o grau em que as notificações estão sendo efetuadas no sistema (Tabela 2). Os resultados demonstram um reconhecimento dos profissionais do sistema de saúde, acerca das subnotificações. Somente $33 \%$ dos profissionais ligados aos hospitais percebe que as intoxicações são diagnosticadas e notificadas. A maioria (56\%) vê apenas uma parte das intoxicações sendo diagnosticadas e notificadas. A visão dos profissionais do sistema municipal e regional de saúde têm percepções mais pessimistas ainda. Tais dados reforçam a percepção de que o Sinan esteja recebendo registros de intoxicações abaixo do número realmente ocorrido.

À medida que os profissionais se inserem na hierarquia superior do sistema, a percepção tende a se tornar mais crítica. Uma explicação pos- sível para essa dissonância pode estar no fato de que a questão analisa indiretamente a conduta e preparo dos profissionais de ponta e o reconhecimento de problemas atestaria contra sua própria atuação profissional. Isso foi mais claro na resposta dos médicos (responsáveis diretos pelos diagnósticos), com menor aceitação de que há equívocos de diagnóstico e de notificação.

Como em todos os níveis do sistema se reconhece a existência de subnotificação, buscouse identificar algumas causas, utilizando-se de hipóteses já aventadas em outros estudos ${ }^{7,28,33}$. Buscou-se contrastar as percepções dos profissionais de ponta e os da hierarquia do sistema de saúde. Em três aspectos ("desconhecimento da equipe quanto à importância do preenchimento das notificações"; "sintomatologia confundida com outras patologias..."; e "falta de capacitação dos profissionais para reconhecer casos de intoxicação...") a percepção entre os profissionais dos hospitais ligados ao atendimento de pacientes, destoaram fortemente dos respondentes na hierarquia superior do sistema. A alta e média hierarquia identificam esses aspectos como causas das subnotificações, em percentuais que variam de 50 a $100 \%$ dos respondentes, conforme a questão e o segmento (Tabela 3), ao passo que

Tabela 1. Comparativo de casos registrados de intoxicação humana por agrotóxico no SINITOX e SINAN, Paraná, 2013 a 2017.

\begin{tabular}{|c|c|c|c|c|c|c|c|c|c|c|}
\hline \multirow{2}{*}{$\begin{array}{c}\text { Ano / } \\
\text { Sistema }\end{array}$} & \multicolumn{2}{|c|}{ Curitiba } & \multicolumn{2}{|c|}{ Londrina } & \multicolumn{2}{|c|}{ Maringá } & \multicolumn{2}{|c|}{ Cascavel } & \multicolumn{2}{|c|}{ Laranjeiras do Sul } \\
\hline & SINITOX & SINAN & SINITOX & SINAN & SINITOX & SINAN & SINITOX & SINAN & SINITOX & SINAN \\
\hline Total & - & 60 & - & 198 & - & 183 & - & 138 & - & 41 \\
\hline 2013 & - & 15 & - & 34 & - & 50 & - & 19 & - & 3 \\
\hline 2014 & - & 10 & - & 48 & & 27 & - & 32 & - & 15 \\
\hline 2015 & - & 7 & - & 33 & - & 19 & - & 34 & - & 12 \\
\hline 2016 & - & 24 & - & 16 & & 46 & - & 27 & - & 3 \\
\hline 2017 & - & 4 & - & 7 & - & 41 & - & 26 & - & 8 \\
\hline
\end{tabular}

Fonte: Adaptado do SINITOX ${ }^{25}$ e SINAN $^{35}$.

Tabela 2. Percepção sobre o grau de diagnóstico e de notificação dos casos de intoxicações por agrotóxicos nas unidades de saúde. Laranjeiras do Sul, Paraná.

\begin{tabular}{lccc}
\hline & \multicolumn{3}{c}{ Locais Pesquisados } \\
\cline { 2 - 4 } \multicolumn{1}{c}{ Afirmativas } & $\begin{array}{c}\mathbf{5}^{\text {a }} \text { Regional de } \\
\text { Saúde }\end{array}$ & $\begin{array}{c}\text { Hospitais: S. Lucas } \\
\text { e S. José }\end{array}$ & $\begin{array}{c}\text { Sec. Munic. } \\
\text { Saúde }\end{array}$ \\
\hline Diagnosticadas e notificadas & $0 \%$ & $33 \%$ & $17 \%$ \\
Parcialmente notificadas e parcialmente notificadas & $50 \%$ & $56 \%$ & $67 \%$ \\
Diagnosticadas, mas não notificadas & $0 \%$ & $0 \%$ & $17 \%$ \\
Dificilmente diagnosticadas & $50 \%$ & $11 \%$ & $0 \%$ \\
\hline
\end{tabular}


Tabela 3. Percepção sobre as causas das subnotificações de intoxicações por agrotóxicos segundo a alocação dos profissionais de saúde. Laranjeiras do Sul e SESA/Paraná.

\begin{tabular}{|c|c|c|c|c|c|}
\hline \multirow[b]{2}{*}{ Causas } & \multicolumn{5}{|c|}{ Locais Pesquisados } \\
\hline & SESA - PR & $\begin{array}{l}5^{\text {a }} \text { Regional } \\
\text { Saúde-PR }\end{array}$ & $\begin{array}{c}\text { Sec. Saúde } \\
\text { Mun. } \\
\text { Laranjeiras } \\
\text { do Sul - PR } \\
\text { (Gestores) }\end{array}$ & $\begin{array}{c}\text { Sec. Saúde } \\
\text { Mun. } \\
\text { Laranjeiras do } \\
\text { Sul - PR (Prof. } \\
\text { Atendimento) }\end{array}$ & $\begin{array}{l}\text { Profissionais } \\
\text { Hospitais } \\
\text { S. Lucas } \\
\text { e S. José - } \\
\text { Laranjeiras } \\
\text { do Sul - PR }\end{array}$ \\
\hline $\begin{array}{l}\text { Desconhecimento da equipe de } \\
\text { saúde quanto a importância do } \\
\text { preenchimento das notificações de } \\
\text { intoxicações nos sistemas oficiais }\end{array}$ & $100 \%$ & $100 \%$ & $100 \%$ & $57,14 \%$ & $14 \%$ \\
\hline $\begin{array}{l}\text { Ausência de exames laboratoriais } \\
\text { conclusivos }\end{array}$ & $0 \%$ & $75 \%$ & $0 \%$ & $14,29 \%$ & $5,26 \%$ \\
\hline $\begin{array}{l}\text { Sintomatologia confundida com } \\
\text { outras patologias comuns em áreas } \\
\text { rurais }\end{array}$ & $50 \%$ & $75 \%$ & $100 \%$ & $14,29 \%$ & $21,05 \%$ \\
\hline $\begin{array}{l}\text { Falta de capacitação para os } \\
\text { profissionais para reconhecer casos } \\
\text { de intoxicação }\end{array}$ & $100 \%$ & $50 \%$ & $50 \%$ & $57,14 \%$ & $21,05 \%$ \\
\hline $\begin{array}{l}\text { Ausência de procedimentos } \\
\text { analíticos mais refinados }\end{array}$ & $0 \%$ & $25 \%$ & $0 \%$ & $14,29 \%$ & $10,53 \%$ \\
\hline $\begin{array}{l}\text { Ausência de adesão a notificação, } \\
\text { pelo tempo consumido no } \\
\text { preenchimento da ficha e pela } \\
\text { ausência de retorno da informação } \\
\text { analisada com as recomendações } \\
\text { técnicas pertinentes }\end{array}$ & $0 \%$ & $50 \%$ & $0 \%$ & $42,86 \%$ & $42,11 \%$ \\
\hline
\end{tabular}

nos hospitais apenas 5 a $21 \%$ dos profissionais concordaram com as mesmas.

Já o item que apresentou maior relevância na percepção dos profissionais de linha de frente (tempo consumido no preenchimento da ficha e ausência de retorno da informação analisada), contraditoriamente foi pouco ou nada citado pelos profissionais da gestão. Na rede de saúde existe um fluxograma instituído, para o feedback dos dados gerados pelas intoxicações. Porém, como muitos dados não estão sendo notificados durante o processo e não se apresentam ações relacionadas aos resultados das notificações, desestimulam-se as ações de saúde pública relacionadas à área. Essa desconexão entre a percepção dos gestores e o segmento de profissionais que realiza atendimento à saúde da população exposta pode ocasionar equívocos de planejamento das ações de saúde pública, como a alocação de esforços onde são menos relevantes e deixando pontos necessários a descoberto.
A pesquisa investigou as intoxicações agudas, classificadas em leves, moderadas e graves (Tabela 4). Nos casos de intoxicações agudas leves, apenas $29 \%$ dos profissionais afirmaram que as mesmas são sempre diagnosticadas conforme o protocolo e em apenas $14 \%$ dos casos se caracteriza a exposição e se estabelece nexo causal, o que confirma a dificuldade de diagnóstico e a existência de subnotificação, visto que são baixos os percentuais de registros de casos e alimentação da base de dados (apenas 57\% afirmam sempre registrar). Associado a esse tema, $89 \%$ dos profissionais alocados na rede hospitalar não passaram por nenhum treinamento relacionado a agrotóxicos no último ano.

No que se refere às intoxicações agudas moderadas e graves, possivelmente devido a maior gravidade do impacto nos pacientes, o grau de subnotificação diminui sensivelmente e se eleva o percentual percebido de caracterização da exposição e da notificação à vigilância. Houve vá- 
Tabela 4. Comparativo da atuação da unidade de saúde nos casos de intoxicação agudas leves, moderadas e graves.

\begin{tabular}{|c|c|c|c|c|c|c|}
\hline \multirow{2}{*}{ Ações } & \multicolumn{3}{|c|}{ Intoxicações agudas leves } & \multicolumn{3}{|c|}{$\begin{array}{c}\text { Intoxicações agudas moderadas e } \\
\text { graves }\end{array}$} \\
\hline & $\begin{array}{l}\text { Não é } \\
\text { realizado }\end{array}$ & Eventualmente & Sempre & $\begin{array}{l}\text { Não é } \\
\text { realizado }\end{array}$ & Eventualmente & Sempre \\
\hline $\begin{array}{l}\text { Diagnosticada conforme o } \\
\text { protocolo }\end{array}$ & $14 \%$ & $57 \%$ & $29 \%$ & $0 \%$ & $57 \%$ & $43 \%$ \\
\hline $\begin{array}{l}\text { Caracteriza a exposição e } \\
\text { estabelecimento nexo causal }\end{array}$ & $29 \%$ & $57 \%$ & $14 \%$ & $0 \%$ & $71 \%$ & $29 \%$ \\
\hline Notifica a vigilância sanitária & $0 \%$ & $43 \%$ & $57 \%$ & $0 \%$ & $29 \%$ & $71 \%$ \\
\hline $\begin{array}{l}\text { Realiza interconsulta com os } \\
\text { centros de informação }\end{array}$ & $43 \%$ & $57 \%$ & $0 \%$ & $43 \%$ & $43 \%$ & $14 \%$ \\
\hline $\begin{array}{l}\text { Registra o caso e alimenta a } \\
\text { base de dados }\end{array}$ & $14 \%$ & $29 \%$ & $57 \%$ & $14 \%$ & $43 \%$ & $43 \%$ \\
\hline
\end{tabular}

Fonte: Tosetto, $2016^{37}$

rios relatos de que pacientes vêm acompanhados por familiares e que muitas vezes trazem a embalagem do agrotóxico causador da intoxicação. Exames complementares raramente são solicitados nos casos de identificação de exposição grave, sendo o paciente imediatamente encaminhado para condução clínica hospitalar. Apenas alguns médicos informaram realizar exames de acetilcolinesterase.

Quanto às intoxicações crônicas, com sintomatologia mais difícil de associar aos agrotóxicos, $43 \%$ dos profissionais de saúde entrevistados afirmam sempre conseguir caracterizara exposição a agrotóxicos, ao passo que $14 \%$ nunca o fazem. A notificação e registro nos sistemas de informação e agravos é feita por $43 \%$ dos profissionais, enquanto $14 \%$ nunca o fazem. Já o estabelecimento do nexo causal da sintomatologia com relação aos agrotóxicos é feita, apenas eventualmente, por $57 \%$ dos profissionais, mas nunca por $28 \%$. Nas intoxicações crônicas, a ausência de procedimentos clínicos e exames laboratoriais conclusivos dificulta a associação à exposição aos agrotóxicos.

Esse conjunto de dados quali e quantitativos confirma hipóteses estabelecidas em vários estudos anteriores sobre a subnotificação das intoxicações por agrotóxicos, mesmo as presentes no Sinan. Os dados lançam luzes sobre aspectoschave para compreender a dinâmica dos sistemas de saúde em relação com a questão e possibilita a indicação de novos rumos para abordar a questão.

\section{Conclusões}

O sistema produtivo do agronegócio brasileiro é baseado na utilização intensiva e abusiva de agrotóxicos que impactam direta e indiretamente no sistema de saúde. Por outro lado, políticas públicas estimulam o uso dessas substâncias ao passo que desconsideram seus efeitos sobre a saúde e meio ambiente. $\mathrm{O}$ estudo aprofundou o olhar sobre a questão das subnotificações das intoxicações desde a perspectiva dos profissionais de saúde e dos sistemas de notificação e informação.

A desatualização de dados do sistema de informações Sinitox e a identificação de subnotificação no lançamento de dados do Sinan confirmou a inconsistência de dados e a falta de comunicação entre eles, resultando no ocultamento da real ocorrência de intoxicações por agrotóxicos. A falta de dados fidedignos dificulta a formulação e implantação de políticas públicas em saúde. O estudo corrobora outros já realizados, concluindo que os sistemas de informação não correspondem adequadamente ao papel de sistemas de vigilância necessitando ser repensados e reconfigurados. Confirmou-se a existência de subnotificações e levantaram-se as possíveis causas relacionadas à dinâmica interna do sistema de saúde. Os profissionais de saúde identificaram situações de falta de preparo adequado para realizar o diagnóstico, falta de tempo, devido a outras demandas do sistema, e/ou falta de pessoal. Foram confirmadas hipóteses de causas apresentadas por diversos pesquisadores como: falta de profissionais treinados para reconhecer quadros de intoxicações, insuficiência de capa- 
citações realizadas no sistema de saúde, que são agravadas pela rotatividade dos profissionais de saúde, especialmente médicos, nos municí$\operatorname{pios}^{7,28,33}$.

Contudo, outras causas foram apontadas: há percepções diferenciadas quanto à problemática, nos diversos níveis do sistema de saúde, ocasionando ruídos no diagnóstico e encaminhamento de ações prioritárias em relação à superação dos problemas da subnotificação; o diagnóstico, a conduta e o tratamento das intoxicações por agrotóxicos não fazem parte do currículo de muitos dos cursos da área de saúde; a capacidade dos serviços de saúde de muitos municípios, aquém da demanda, ocasiona sobrecarga de trabalho aos profissionais e favorece a subnotificação das intoxicações por agrotóxicos; a população exposta a agrotóxicos nem sempre é identificada quando procura atendimento na unidade de saúde, o que dificulta a correlação agravos de saúde e agrotóxicos.

O estudo compactua com a ideia da Associação Brasileira de Saúde Coletiva (ABRASCO) de que é preciso fortalecer e criar espaços na agenda política e financeira do SUS, para a estruturação da vigilância, atenção e promoção da saúde rela- cionada a agrotóxicos. Além disso, a articulação intersetorial e a adoção de políticas de incentivo à Agroecologia são fundamentais para que o modelo agrícola seja alterado ${ }^{34}$. Por último, são apresentadas sugestões, tanto dos entrevistados como dos autores, a partir do estudo, para redução das subnotificações de intoxicação por agrotóxicos: revisão dos protocolos e fluxos de informações entre as unidades de saúde e os sistemas de informação toxicológica, tendo em vista o feedback a todo o sistema, das informações lançadas e das medidas tomadas; capacitação permanente dos profissionais de saúde, acerca do diagnóstico, notificação e tratamento adequado em intoxicação por agrotóxicos, especialmente em regiões agrícolas; estabelecimento de protocolo simplificado para notificação de intoxicação por agrotóxicos, tanto para o pessoal do sistema de saúde como para outras pessoas relacionadas à atividade; realização de campanhas e ações específicas de conscientização, voltada a agricultores e ao sistema de saúde, considerando os ciclos agrícolas; realização de capacitações de agricultores e pessoal de comércio de agrotóxicos acerca dos riscos e boas práticas na comercialização e uso dos mesmos.

\section{Colaboradores}

EE Tosetto produziu a dissertação de mestrado em Agroecologia e desenvolvimento rural sustentável, o qual deu base para o artigo. Apoiou desde o planejamento da pesquisa e na coleta de dados a campo, bem como nas análises dos dados, assim como na redação do artigo. AI Andrioli orientou o trabalho, participou da interpretação e análise dos dados obtidos e na redação do artigo. PI Christoffoli coorientou o trabalho, apoiou no planejamento do estudo, orientou a coleta de dados a campo e parte das análises de dados, bem como apoiou na análise dos dados e redação do artigo. 


\section{Referências}

1. Delgado GC. Do capital financeiro na agricultura à economia do agronegócio. Porto Alegre: EDUFRGS; 2012.

2. Londres F. Agrotóxicos no Brasil: um guia para ação em defesa da vida. Rio de Janeiro: ASPTA - Assessoria e Serviços a Projetos em Agricultura Alternativa, 2011.

3. Porto MF, Soares WL. Modelo de desenvolvimento, agrotóxicos e saúde: um panorama da realidade agrícola brasileira e propostas para uma agenda de pesquisa inovadora. Rev Bras Saude Ocup 2012; 37(125):17-50.

4. Rigotto RM, Aguiar ACP. Invisibilidade ou invisibilização dos efeitos crônicos dos agrotóxicos à saúde? Desafios à ciência e às políticas públicas. In: Observatório Internacional de Capacidades Humanas, Desenvolvimento e Políticas Públicas: estudos e análises. Brasília: UnB/ObservaRH/Nesp, Fiocruz/Nethis; 2015.

5. Moreira JC, Peres F, Simões AC, Pignati WA, Dores EC, Vieira SN, Strüssmann C, Mott T. Contaminação de águas superficiais e de chuva por agrotóxicos em uma região do estado do Mato Grosso. Cien Saude Colet 2012; 17(6):1557-1568.

6. Bombardi LM. Um atlas de nossa agricultura envenenada. 2015. [acessado: 2016 jul. 13]. [acessado 2020 maio 28]. Disponível em: http://outraspalavras.net/ brasil/um-atlas-de-nossa-agricultura-envenenada.

7. Peres F, Moreira JC, organizadores. É veneno ou é remédio? Agrotóxicos, saúde e ambiente uma introdução ao tema. Rio de Janeiro: Editora Fiocruz; 2003.

8. Secretaria de Saúde do Estado do Paraná (SESA). Intoxicações Agudas Por Agrotóxicos. Atendimento Inicial do Paciente Intoxicado; 2018. [acessado 2020 maio 1]. Disponível em: https://www.saude.pr.gov.br/sites/ default/arquivos_restritos/files/documento/2020-04/ intoxicacoesagudasagrotoxicos2018.pdf

9. International Agency for Research on Cancer (IARC). Some Organophosphate Insecticides and Herbicides. IARC Monographs on the Evaluation of Carcinogenic Risks to Humans. Vol 112. Lyon: IARC; 2015. [cited 2020 maio 5]. Available from: https://publications. iarc.fr/549.

10. Instituto Brasileiro de Geografia e Estatística (IBGE). Censo agropecuário: resultados definitivos 2017. Rio de Janeiro: IBGE; 2019

11. Sistema de Monitoramento do Comércio e Uso de Agrotóxicos do Paraná (Siagro). Secretaria de Estado da Agricultura e do Abastecimento (Seab). Consumo de agrotóxicos por município. 2018. UFPR, Portal Saúde. [acessado 2020 maio 1]. Disponível: http://www. saude.ufpr.br/portal/observatorio/wp-content/uploads/sites/12/2015/11/SIAGRO_Com\%C3\%A9rcio_ Uso_agrot\%C3\%B3xicos_Paran\%C3\%A1_2013_ 2018-1.pdf.

12. Brasil. Companhia Nacional de Abastecimento (Conab). Acompanhamento da Safra Brasileira de Grãos. Vol. 5, Safra 2017/2018, n. 12. Setembro/2018. [acessado 2019 nov 14]. Disponível em: https://www.conab.gov.br/info-agro/safras/graos/boletim-da-safrade-graos.

13. Presa RB, Christoffoli PI. Crédito rural cooperativo e desenvolvimento local. Estudo de caso no município de Laranjeiras do Sul (PR). In: Gomes IA. A Geografia na contemporaneidade. Ponta Grossa: Atena; 2018.
14. Sistema de Comércio e Uso de Agrotóxicos no Estado do Paraná (Siagro). Volume de Agrotóxicos Comercializados - 2013 a 2018. [acessado 2019 nov 19]. Disponível em: http://www.adapar.pr.gov.br/.

15. Martins GF. Relatório: Vigilância em saúde de populações exposta a agrotóxicos no Estado do Paraná. Brasília: Ministério da Saúde; 2015. [acessado 2019 nov 5]. Disponível: http://www.saude.gov.br/images/ pdf/2015/julho/08/Relatorio-Paran--.pdf.

16. Tavella LB, Silva IN, Fontes LO, Dias JRM, Silva MIL. Uso de agrotóxicos na agricultura e suas consequências toxicológicas e ambientais. Agropecuária Científica no Semi Árido 2011; 7(2):6-12.

17. Neves PDM, Bellini M. Intoxicações por agrotóxicos na mesorregião norte central paranaense, Brasil 2002 a 2011. Cien Saude Colet 2013; 18(11):31-55.

18. Machado MB. Associação entre exposição a agrotóxicos, depressão e desesperança na população do município de Anahy/PR [dissertação]. Cascavel: Universidade Estadual do Oeste do Paraná; 2018.

19. Brasil. Ministério da Saúde (MS). Secretaria de Atenção à Saúde. Protocolo de atenção à saúde dos trabalhadores expostos a agrotóxicos. Brasília: MS; 2006. [acessado: 2014 mai. 08]. Disponível em: http:// bvsms.saude.gov.br/bvs/publicacoes/protocolo_atencao_saude_trab_exp_agrotoxicos.pdf.

20. Silva JM, Silva EM, Faria HP, Pinheiro TMM. Agrotóxico e trabalho: uma combinação perigosa para a saúde do trabalhador rural. Cien Saude Colet 2005; 10(4):891-903.

21. Faria NMX, Fassa AG, Facchini LA. Intoxicação por agrotóxicos no Brasil: os sistemas oficiais de informação e desafios para realização de estudos epidemiológicos. Cien Saude Colet 2007; 12(1):25-38.

22. Rebello FM, Caldas ED, Heliodoro VO, Rebello RM. Intoxicação por agrotóxicos no Distrito Federal, Brasil, de 2004 a 2007: análise da notificação ao Centro de Informação e Assistência Toxicológica. Cien Saude Colet 2011; 16(8):3493-3502.

23. Brasil. Agência Nacional de Vigilância Sanitária (Anvisa). Critérios para a classificação Toxicológica. [acessado 2016 abr 25]. Disponível em: http://goo.gl/6Ce$8 \mathrm{VY}$.

24. Brasil. Ministério da Saúde (MS). Portaria $n^{\circ} 204$, de 17 de fevereiro de 2016. Brasília: MS; 2016. [acessado 2020 maio 11]. Disponível em: https:// bvsms.saude.gov.br/bvs/saudelegis/gm/2016/ prt0204_17_02_2016.html

25. Fundação Oswaldo Cruz (Fiocruz). Sistema Nacional de Informações Tóxico Farmacológicas - SINITOX. [acessado 2019 nov 5]. Disponível em: https://sinitox. icict.fiocruz.br/.

26. Bochner R. Sistema Nacional Informações Tóxico Farmacológicas - SINITOX e as intoxicações humanas por agrotóxicos no Brasil. Cien Saude Colet 2007; 12(1):73-89.

27. Organização Pan Americana da Saúde (OPAS). Manual de Vigilância de Saúde da População Exposta a Agrotóxicos. 1996. [acessado 2016 abr 7]. Disponível em: https://www.paho.org/bra/index.php?option=com _docman\&view=document\&category_slug=saude -e-ambiente-707\&alias=301-manual-vigilancia-dasaude-populacoes-expostas-a-agrotoxicos-1\&Itemid $=965$. 
28. Secretaria de Saúde do Estado do Paraná (SESA). Plano Diretor de regionalização: hierarquização e regionalização da assistência à saúde, no estado do Paraná. São Paulo: SESA; 2013. [acessado $2014 \mathrm{dez} 18$ ]. Disponível em: http://www.saude.pr.gov.br/arquivos/ File/PDR_atualizado__Edson.pdf.

29. Peres F, Silva JJO, Della-Rosa HV Lucca SR. Desafios ao estudo da contaminação humana e ambiental por agrotóxicos. Cien Saude Colet 2005; 10 (Supl.):27-37.

30. Dias MDA, Bertolini GCS, Pimenta AL. Saúde do trabalhador na atenção básica: Análise a partir de uma experiência municipal. Trab Educ Saude 2011; 9(1):137-148.

31. Menck VF, Serafim MP, Oliveira JM. (2019). Intoxicação do(a) trabalhador(a) rural por agrotóxicos: (sub) notificação e (in)visibilidade nas políticas públicas de 2001 a 2015. Segurança Alimentar e Nutricional 2019; 26:e019001.

32. Pigou AC, Ramos FS. La Economia Del Bienestar. Editora Madrid: M. Aguilar; 1946.

33. Veigas APB, Carmo RF, Luz ZMP. Fatores que influenciam o acesso aos serviços de saúde na visão de profissionais e usuários de uma unidade básica de referência. Saude Soc 2015; 4(1):100 -112.

34. Carneiro FF, Pignati W, Rigotto RM, Augusto LGS, Rizzolo A, Faria NM, Alexandre VP, Friedrich K, Mello MSC. Um alerta sobre os impactos dos agrotóxicos na saúde. Dossiê Abrasco. Parte 1. Agrotóxicos, Segurança Alimentar e Nutricional e Saúde. Rio de Janeiro: ABRASCO; 2012.

35. Sistema de Informação de Agravos de Notificação (Sinan). [acessado 2020 jun 20]. Disponível em: sinanhttps://portalsinan.saude.gov.br/

36. Brasil. Ministério da Saúde (MS). SIHSUS. Sistema de Informação Sobre Mortalidade (SIM). [acessado 2016 abr 16]. Disponível em: http://svs.aids.gov.br/ cgiae/sim/.

37. Tosetto EE. Identificação das causas das subnotificações das intoxicações da população exposta a agrotóxicos: estudo de caso de Laranjeiras do Sul - PR [dissertação]. Laranjeiras do Sul: Universidade Federal da Fronteira Sul; 2016.

Artigo apresentado em 23/06/2020

Aprovado em 26/07/2021

Versão final apresentada em 28/07/2021

Editores-chefes: Romeu Gomes, Antônio Augusto Moura da Silva 
